LIAMES 12 - pp. 67-85, Primavera 2012

Lilián Guerrero

(Universidad Nacional Autónoma de México, México)

\title{
La naturaleza de las frases locativas en construcciones de movimiento en yaqui
}

\begin{abstract}
This article examines the spatial relations associated to motion constructions in Yaqui (Uto-Aztecan, Mexico). The aim of this paper is to examine the formal codification of place, source and goal notions, as well as the possible combinations between verb types and types of locative phrases. It is shown that Yaqui neutralizes the formal distinction between place and source, a very uncommon pattern in languages of the world. Based on the combinations found, two subtypes of manner of motion predicates are established, in terms of their (in)compatibility with phrases expressing goal or source (i.e., telic locative phrases).

KEYWORDS: Motion verbs; Locative phrases; Satellite-frame; Yaqui.

RESUMEN: Este artículo explora la naturaleza de las relaciones espaciales asociadas a construcciones de movimiento en la lengua yaqui (yutoazteca, México), con el fin de indagar la codificación formal de las nociones de lugar, fuente y meta, así como las posibles combinaciones entre los tipos de verbos y los tipos de locación. Los resultados de este estudio muestran que el yaqui neutraliza la distinción entre fuente y locación, un patrón muy poco frecuente en las lenguas del mundo. Además, a partir de las asociaciones encontradas en corpus, se distinguen dos subgrupos de verbos de manera de movimiento dada su (in)compatibilidad con frases locativas dinámicas que expresan meta u origen (i.e. trayectorias télicas).

PALABRAS CLAVES: Verbos de movimiento; Frases locativas; Marco satelital; Yaqui.
\end{abstract}

\section{INTRODUCCIÓN ${ }^{1}$}

En este artículo se explora la estructura argumental de construcciones de movimiento en la lengua yaqui (yutoazteca, México). Los objetivos son, primero, examinar los recursos de codificación formal de las relaciones espaciales y, segundo, explorar las asociaciones de tipos de locación con los tipos de eventos, a partir de un análisis de corpus. Aunque la expresión de lugar, meta y fuente es compatible con casi cualquier tipo de evento (ej. vi a María en el parque desde mi ventana), los verbos de movimiento y posición no solo tienden a co-ocurrir con fases locativas, sino que también muestran ciertas preferencia de combinación a partir de su semántica.

\footnotetext{
${ }^{1}$ Agradezco sinceramente los atinados comentarios sobre este artículo de dos lectores anónimos de la Revista LIAMES; cualquier error es mi entera responsabilidad. Esta investigación ha sido financiada en parte por el proyecto CONACyT (No. 83529) y forma parte de un estudio mayor que busca explorar el estatus gramatical y discursivo de los complementos locativos asociados a movimiento y posición en lenguas yutoaztecas. Para un primer acercamiento a la interfaz sintaxis-pragmática en yaqui, véase Belloro y Guerrero (2012), y en el náhuatl, Guerrero y Belloro (en prep.).
} 
El presente análisis parte del supuesto de que la interpretación de la oración se construye no solo de los componentes semánticos sino también de la codificación morfosintáctica de los constituyentes de la oración. En yaqui, la distinción entre verbos de movimiento y algunos verbos de manera de movimiento no es tajante, al menos no en lo que respecta a su asociación con frases locativas que expresan la dirección del movimiento. Además, la marcación formal de las relaciones espaciales en esta lengua refleja uno de los patrones tipológicamente menos frecuentes (Pantcheva 2010), pues neutraliza la distinción entre fuente y lugar, i.e. sincretismo morfológico.

En lo que sigue, se introduce brevemente la tipología del movimiento y locación; en $§ 3$ se describen las propiedades morfosintácticas básicas del yaqui y la codificación de expresiones locativas; en $\S 4$ se analizan las distintas combinaciones de locación y tipos de verbos de movimiento. A manera de conclusión, en $§ 5$ se discute el comportamiento de los predicados en yaqui y se anticipan algunas reflexiones finales. El yaqui pertenece al subgrupo taracahita de la familia yutoazteca (rama sureña); se habla en el sur de Sonora (noroeste de México) por alrededor de 15,000 hablantes, y en el sur de Arizona (Estados Unidos) por cerca de 6,000 hablantes. Los datos que aquí se analizan provienen de la variante sonorense.

\section{LA TIPOLOGÍA DEL MOVIMIENTOY LA LOCACIÓN}

El estudio sobre verbos de movimiento ha sido sumamente fructífero desde la propuesta original de Tesnière (1959) de dos grupos de verbos, mouvement y déplacement; no obstante, es la tipología de Talmy (1985; 1991; 2000) y las revisiones posteriores (Slobin 1996, 2004) las que más impacto han tenido en la descripción de este tipo de eventos en lenguas particulares. Según Talmy, el esquema conceptual de movimiento básico se conceptualiza a partir de la entidad que se mueve o está dispuesta en cierta postura (figura), el movimiento o locación de la figura, y la base o fondo con respecto a la cual se mueve la figura o se caracteriza su situación locativa. Otros componentes incluyen la trayectoria (la dirección que sigue la figura), la manera y la causa potencial del movimiento.

En las lenguas del mundo, las raíces verbales suelen lexicalizar algunos de estos componentes semánticos, en especial la manera y/o la trayectoria. A partir del patrón de lexicalización predominante, se proponen distintos tipos de lenguas. Así, en lenguas de marco-V (verbales) como las romances y semíticas, turco, nez perce, tamil, japonés y coreano, la raíz verbal lexicaliza la trayectoria, ej. la botella entró a la cueva flotando. En lenguas de marco-S (satélites) como el inglés, chino, warlpiri, ojibwa, la raíz verbal lexicaliza la manera, ej. the bottled floated into the cave. Si el predicado lexicaliza la trayectoria (marco-V), entonces la manera puede expresarse vía una frase o cláusula periférica (ej. gerundio); si la trayectoria no se lexicaliza en el verbo (marco-S), entonces puede expresarse de manera 'satelital' a través de partículas o afijos verbales. ${ }^{2}$ La relación entre los componentes semánticos y sus manifestaciones morfosintácticas no suele ser unívoca y, generalmente, co-existen más de

\footnotetext{
2 Aunque con menor frecuencia, se encuentran también lenguas del tipo-F en donde el verbo lexicaliza algunas características de la figura (ej. atsugewi). No se conocen lenguas en donde la lexicalización de la base sea el patrón predominante, aunque algunas lenguas dan cuenta de ciertas raíces, ej. verbos que lexicalizan el locatum como 'enjaular' o 'embotellar'.
} 
un patrón en una misma lengua. Por este motivo, es preferible establecer la descripción en términos de construcciones, más que de lenguas.

En relación con las frases locativas asociadas al movimiento, destacan dos tipos de información: el dominio en donde la figura está localizada en relación con el fondo (generalmente codificada con marcas de caso locativo o adposiciones locativas) y el rol semántico del dominio espacial (Nikitina 2009: 1114). Este último puede ser dinámico, si la figura está en movimiento, o estático, si se predice el lugar o ubicación de la figura con respecto al fondo (Tabla 1). Una relación dinámica generalmente codifica trayectoria, ej. meta, fuente, rumbo, dirección, mientras que una relación estática expresa la ubicación de la figura con respecto a la base, ej. lugar.

Tabla 1. Relaciones espaciales locativas (adaptado de Nikitina 2009)

\begin{tabular}{ccc}
\hline relación espacial & rol semántico & tipos de trayectoria \\
\hline dinámico & meta & télica (delimitada) \\
& fuente & télica (delimitada) \\
estático & lugar & atélica (no delimitada) \\
\hline
\end{tabular}

Siguiendo la propuesta de Aske (1989), es posible distinguir entre las relaciones espaciales dinámicas, frases que describen trayectorias télicas, esto es, puntos culminantes del movimiento, y frases que describen trayectorias atélicas, es decir, expresiones que refieren a la ruta, vía u orientación del movimiento. En esa clasificación, las locaciones estáticas se consideran trayectorias atélicas, pues solo las primeras involucran un estado resultativo, i. e. el cambio de lugar de la figura, pero las estáticas describen la ubicación donde se lleva a cabo el evento, como un todo. En español, la trayectoria de destino suele codificarse con la preposición a, ej. Juan se fue a la biblioteca, mientras que la trayectoria de origen se marca con de, ej. Juan salió de la biblioteca; las rutas y vías (trayectorias atélicas) suelen hacer uso de otras preposiciones, ej. Atravesó la ciudad vía/por Insurgentes, caminó hasta su casa.

Todas las lenguas tienen algún recurso para codificar lugar, meta y fuente del movimiento, pero no todas tienen un paradigma de marcas de caso o marcas léxicas (adposiciones) amplio, ni todas las lenguas distinguen formalmente las tres nociones. En un estudio tipológico reciente sobre la codificación formal de estas relaciones espaciales fuente, meta y lugar, Pantcheva (2010: 1043-4) examina la representación de los cinco patrones posibles de codificación en lenguas del mundo (ejemplos tomados del original):

\footnotetext{
a. lugar $=$ meta $=$ fuente; una sola marca codifica las tres relaciones espaciales; ej. en maya yucateco ich le-kaaha-o 'en / a / fuera de la caja' (Bohnemeyer y Pérez Báez 2008)

b. lugar $=$ meta $\neq$ fuente , una marca expresa lugar y meta, otra marca codifica la fuente; ej. en bretón war an daol 'en/hacia la mesa', diwar an daol 'de la mesa' (Ternes 1992)

c. lugar $\neq$ meta $\neq$ fuente; una marca independiente para cada relación espacial; ej. en indonesio, di Australia 'en Australia', ke Johor 'a/hacia Johor', dari Malaysia 'desde Malasia' (Mintz 1994)
} 
d. lugar $=$ fuente $\neq$ meta ; las nociones de lugar y fuente comparten la marca, mientras que la meta hace uso de una segunda marca

e. lugar $\neq$ meta $=$ fuente; una misma marca codifica meta y fuente, pero se diferencia lugar.

En la muestra tipológica de Pantcheva, y en concordancia con los resultados de Blake (1997) para lenguas australianas, Noonan (2008) lenguas tibeto-burmanas, y la muestra tipológica de Rice y Kabata (2007), se encuentran solo tres de las cinco combinaciones: la distinción formal de cada relación espacial (1c), como el patrón más común, el sincretismo entre meta y fuente (1b) y, por último, la neutralización formal de las tres nociones (1c).

Tabla 2. Patrones de sincretismo en la lexicalización de lugar (L), meta (M), y fuente (F) (adaptado de Pantcheva 2010: 1046)

\begin{tabular}{lcccc}
\hline & Blake & Noonan & Rice y Kabata & Pantcheva \\
\hline $\mathrm{L}=\mathrm{M}=\mathrm{F}$ & $0 \%(0 / 85)$ & $4 \%(3 / 76)$ & $11 \%(5 / 44)$ & $13 \%(7 / 53)$ \\
$\mathrm{L}=\mathrm{M} \neq \mathrm{F}$ & $9 \%(8 / 85)$ & $58 \%(44 / 76)$ & $23 \%(10 / 44)$ & $34 \%(18 / 53)$ \\
$L \neq M \neq F$ & $91 \%(71 / 85)$ & $33 \%(0 / 76)$ & - & $53 \%(28 / 53)$ \\
$\mathrm{L}=\mathrm{F} \neq \mathrm{M}$ & $0 \%(0 / 85)$ & $2.5 \%(2 / 76)$ & - & $0 \%(0 / 53)$ \\
$\mathrm{L} \neq \mathrm{M}=\mathrm{F}$ & $0 \%(0 / 85)$ & $2.5 \%(2 / 76)$ & $0 \%(0 / 44)$ & $0 \%(0 / 53)$ \\
\hline
\end{tabular}

Esto es, de los cinco posibles patrones de sincretismo (1), solo tres se documentan en las lenguas del mundo, mientras que los otros dos apenas se documentan en un par de lenguas tibeto-burmanas: el sincretismo entre lugar y fuente (1d) y el de meta y fuente (1e). Veremos que el yaqui muestra uno de los patrones menos esperados, al neutralizar la distinción entre lugar y fuente.

Finalmente, con respecto a las combinaciones esperadas entre tipos de eventos de movimiento y tipos de relaciones espaciales, autores como Morimoto (2007: 288-9) han mostrado que las raíces que perfilan la manera de movimiento $\left(\mathrm{MOVE}_{\mathrm{M}}\right)$ son perfectamente compatibles con frases dinámicas (télicas) en lenguas/construcciones de marco-S, ej. She danced into the kitchen, John swam to the island, mientras que este tipo de combinaciones son menos afortunadas en lenguas/construcciones de marco-V, ej. \#Ella bailó a la cocina, \#Juan nadó a la isla, ${ }^{3}$ pues este tipo de descripciones prefiere asociarse a locaciones estáticas, ej. Bailó en la cocina, nadó en mar abierto. En cambio, las raíces $\operatorname{Move}_{\mathrm{T}}$ (aquellas que lexicalizan la trayectoria) se asocian, de manera natural, a

\footnotetext{
${ }^{3}$ Algunas raíces $\operatorname{MOVE}_{M}$ pueden asociarse a una frase direccional; estas combinaciones suelen marcarse con preposiciones no-télicas como en nadó hasta la orilla, caminó hacia la biblioteca. Véase Aske (1989) y Cuartero (2010) para una discusión detallada sobre estos dos tipos de trayectorias y las múltiples funciones de las preposiciones en español. Las aportaciones de Lamiroy (1991), Cifuentes Honrubia (1999), Crego García (2000), Ibáñez (2005), son solo algunos de los muchos que abordan los verbos de movimiento en español; véase también los trabajos comparativos en Ibarrexte (2004), Morimoto $(2000,2007)$ y Cifuentes Férez (2009), así como el estudio tipológico de Beavers, Levin y Tham (2010).
} 
predicados que lexicalizan una dirección inherentemente télica, ej. Juan llegó a la biblioteca. Para lenguas/construcciones de marco-S, se dice que solo tienen un tipo de raíces verbales (Beavers, Levin y Tham, 2010) de tal forma que todos los verbos MOVE $_{\mathrm{M}}$ pueden usarse en una construcción de movimiento dirigido al combinarse con frases dinámicas télicas, ej. Pat ran / walked / ambled / jogged to the store, mientras que las combinaciones con locaciones estáticas son pragmáticamente más restringidas, ej. \#Pat ran in the store.

En trabajos posteriores, Slobin encuentra otras diferencias importantes entre los dos grandes tipos de patrones de lexicalización, a partir de las expresiones preferidas en narraciones discursivas: (i) las lenguas de marco-S tienen un léxico de movimiento más expresivo y amplio, especialmente para codificar manera del movimiento, en comparación con las de marco-V; y (ii) describen la base (locación) más frecuentemente y de manera más elaborada que las lenguas de marco- $\mathrm{V}$, porque sus características sintácticas permiten combinar satélites y frases locativas en una misma cláusula.

Como se mostrará más adelante, el yaqui puede caracterizarse como una lengua de marco-S ya que las raíces verbales lexicalizan, preferentemente, el movimiento y la manera como se mueve la figura, mientras que la trayectoria se expresa fuera del verbo, ya sea mediante satélites, frases locativas o ambos. El análisis parte de un corpus de casi 650 construcciones obtenidas de textos orales, del diccionario yaqui-español (Estrada et al. 2004), y del trabajo de campo.

Tabla 3. Corpus de construcciones de movimiento en yaqui

\begin{tabular}{lr}
\hline Textos orales & 190 \\
notas de campo (directos, cuestionarios) & 122 \\
Diccionario (Estrada et al 2004) & 334 \\
& 646 cláusulas \\
\hline
\end{tabular}

Entre los estudios preliminares sobre verbos de movimiento y posición en yaqui, se incluyen aquellos que exploran algunas propiedades de sus componentes semánticos, ej. supleción de las formas verbales a partir de la naturaleza de la figura (Guerrero 2004a); los tipos aspectuales de predicados y la propuesta de intransitividad escindida (Guerrero 2004b), y la correlación que existe entre la propiedades de la figura y la codificación de verbos de postura (Gutiérrez 2011). Se ha iniciado también al estudio de la interfaz sintaxisdiscurso de los complementos locativos en cláusulas de movimiento (Belloro y Guerrero 2012, Guerrero y Belloro, en prep.). Este es el primer acercamiento formal a la codificación de las relaciones locativas y las asociaciones entre los tipos de raíces verbales y relaciones espaciales.

\section{LA LENGUA YAQUI}

El yaqui es una lengua sintética-aglutinante, con un marco casual nominativo-acusativo. Los argumentos léxicos acusativos aparecen marcados por el sufijo -ta y los nominativos no se marcan (2a); los argumentos oblicuos se marcan con adposiciones (2b). El sufijo acusativo 
-ta y el plural -(i)m son mutuamente excluyentes (2c). Como lengua de núcleo final, el yaqui hace uso exclusivo de sufijos y posposiciones, en especial para codificar relaciones semánticas como benefactivos, concomitantes, instrumentos y relaciones espaciales. ${ }^{4}$

(2)
a.
$\begin{array}{ll}\text { Wa'a- } \varnothing & \text { rancheo- } \varnothing \\ \text { DEM-NOM } & \text { granjero-NOM } \\ \text { 'Este granjero encontró una vaca' }\end{array}$
wakas-ta
ta'aru-k
perder-PFV
'Este granjero encontró una vaca.'
b.

$\begin{array}{llll}\text { Aapo } & \text { u-e } & \text { wakas-ta- } u & \text { biute- } k \\ \text { 3SG.NOM } & \text { DET-OBL } & \text { vaca-ACC-DIR } & \text { silbar-PFV } \\ \text { 'Él le silbó a la vaca.' } & & \end{array}$

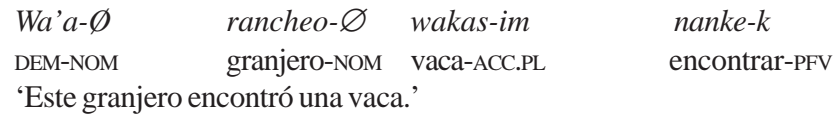
d. Aapo am= nanke- $k$
3SG.NOM 3PL.ACC $=$ encontrar-PFV
'Él las encontró.'

Para el nominativo y acusativo, hay dos series de pronombres, tradicionalmente llamados "plenos" y "reducidos" (Tabla 4); los primeros muestran una distribución análoga a la de las frases léxicas como aapo en $(2 \mathrm{~b}, \mathrm{~d})$, mientras que los segundos tienen el estatus morfo-fonológico de clíticos; las terceras personas carecen de pronombres reducidos para el nominativo, pero son las únicas que presentan proclíticos acusativos (2d). Los pronombres que acompañan a las posposiciones tienen su propio paradigma (pronombres oblicuos).

Tabla 4. Sistema pronominal

\begin{tabular}{|c|c|c|c|c|}
\hline & nominativo & acusativo & oblicuo & genitivo \\
\hline $1 \mathrm{Sg}$ & inepo $=n e$ & nee & ne & in, nim \\
\hline & inepo ='e & enchi & $e-$ & em \\
\hline $3 \mathrm{Sg}$ & aаpo & apo'ik $a=$ & $a-$ & a-/aapo'ik \\
\hline $1 \mathrm{P} 1$ & itepo $=$ te & itom & ito- & itom \\
\hline $2 \mathrm{PI}$ & eme'e ='em & enchim & emo- & em, enchim \\
\hline $3 \mathrm{P} 1$ & bempo $=m e$ & apo'im am= & ame- & bem, beтро'im \\
\hline
\end{tabular}

${ }^{4}$ Abreviaturas: ACC: acusativo, DESID: desiderativo, DEM: demonstrativo, DET: determinante, DIR: direccional, GEN: genitivo, IMPFV: imperfectivo, LOC: locativo, MD: marca discursiva, NEG: negación, NMLZ: nominalizador, NOM: nominativo, oBL: oblicuo, PAS: pasado, Рот: potencial, PFV: perfectivo, PASs: pasivo, PL: plural, PRE: presente, SG: singular. 
El yaqui tiene un orden de constituyentes fijo SOV. Los arreglos lineales alternativos más frecuentes corresponden a dislocaciones a la derecha del participante tipo sujeto (3a) u objeto (3b), con la función pragmática de antitópico (Belloro y Guerrero 2010).

(3) a.

$$
\begin{array}{llll}
\text { Junaman } & \text { itom }=\text { to'o- } b \text { wite- } k & \text { juna'a } & \text { Mayor- } \varnothing \\
\text { allí } & \text { 1PL.ACC }=\text { dejar-correr-PFV } & \text { DEM } & \text { Mayor-NOM }
\end{array}
$$
'Nos abandonó allí, el Mayor.' (Silva 2004, HVH: 39)

b

$$
\begin{array}{llcl}
\text { Jiba } & a_{i}=\text { bitchu- } k & u-k a \text { 'a } & \text { jamut-ta } \\
\text { siempre } & \text { 3SG.ACC }=\text { mirar-PFV } & \text { DET-ACC } & \text { mujer-ACC } \\
\text { 'Siempre la miraba, a la mujer.' (Jiak nokpo's 2: 26) } &
\end{array}
$$

c.

$$
\begin{aligned}
& \text { Junak bea ne in chiva-m batwe-po to'o-siika } \\
& \text { entonces MD 1SG.NOM 1sG.GEN chiva-PL río-LOC dejar-ir.SG.PFV } \\
& \text { jo'ara-u-bicha siika } \\
& \text { casa-DIR-HACIA ir.SG.PFV (Buitimea 2007; viejita viuda:4) }
\end{aligned}
$$

Las relaciones espaciales locativas pueden hacer uso de deícticos espaciales o pro-adverbios, como junaman 'allí' en (3a), frases posposicionales como en (3c), o ambos. A diferencia de las frases locativas, los pro-adverbios suelen mantener una posición fija dentro de la construcción, pues siempre anteceden al predicado. El paradigma de posposiciones locativas se lista en la Tabla 5. Algunas posposiciones son libres, otras son ligadas; algunas suplen en número. Del paradigma, las más frecuentes en corpus para la codificación de frases direccionales (dinámicas) y de locación (estáticas) son - $u$, - po, y -bicha.

Tabla 5. Posposiciones locativas

\begin{tabular}{llll}
\hline -u,-wi/-meu,-mewi & 'a (sg/pl)' & -po & 'en, de, desde' \\
-bicha & 'hacia' & -t, -chi/-met & 'en, sobre (sg/pl)' \\
luula & 'hacia, derecho' & -betana & 'de, desde' \\
naapo & 'cerca' & beas & 'enfrente' \\
-ku & 'en medio' & bepa & 'sobre, arriba' \\
nasuk & 'entre' & betuk & 'debajo' \\
\hline
\end{tabular}

El punto final de una actividad, incluyendo el punto de llegada de los verbos de movimiento, se introduce con la posposición direccional $-u$ (4a), mientras que el punto transversal (ruta, rumbo) se marca con la posposición -bicha 'hacia' o lula 'derecho, línea recta'. Estas últimas marcas suelen acompañarse de otras frases locativas, sean deícticos u otras frases posposicionales, pero nunca aparecen por sí solas (4b), i.e., posposiciones compuestas como enfrente de, a espaldas de. Para facilitar la lectura, en los ejemplos las frases locativas aparecen entre corchetes. 


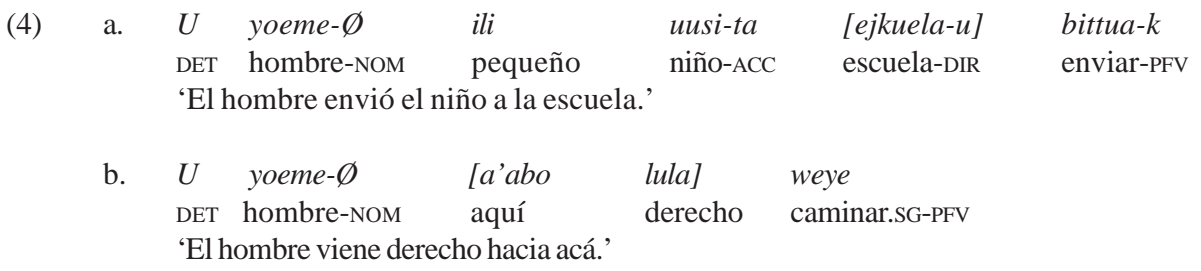

En yaqui, una frase locativa marcada únicamente con el direccional - $u$ describe una trayectoria télica, esto es, explicita el punto final del movimiento y el cambio de locación de la figura. Eso explica porqué la oración en (5a) se desfavorece, i.e. debe haber un cambio de lugar. En cambio, aquellas frases marcadas con -bicha y lula introducen trayectorias atélicas, es decir, expresan la dirección, tránsito, la vía por donde se lleva a cabo el movimiento, pero no la culminación ni el cambio de lugar de la entidad que se mueve (5b). ${ }^{5}$

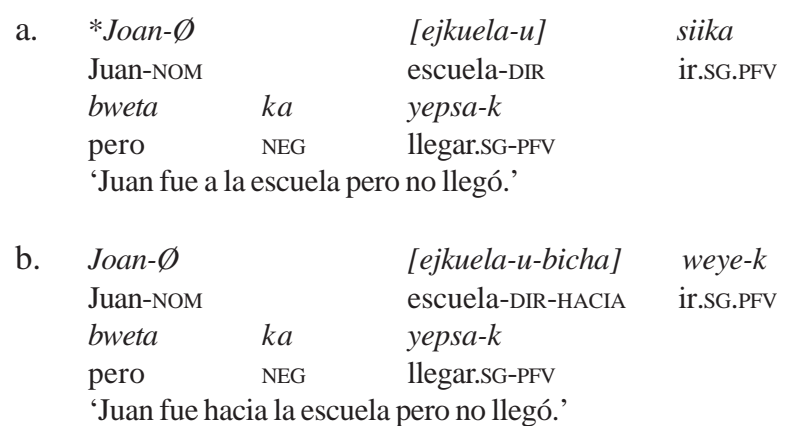

La posposición - po codifica ubicación física (6a-b) y temporal (6c); por su naturaleza, una frase locativa estática marcada con -po puede combinarse con cualquier tipo de predicados incluidos estativos, procesos de cambio y causativos (6c), además de verbos de movimiento, i.e. ubica el evento en un espacio delimitado.
a.
Junak
bea $=$ ne
$\mathrm{MD}=1 \mathrm{SG} . \mathrm{NOM}$
[koloso-po] jo'a-ka-me
Coloso-LOC vivir-PAS-NMLZ
in wawai-m-meu yepsa-k,
1SG.POS pariente-PL-DIR llegar.SG-PFV
'Luego llegué con mis parientes que vivían en el Coloso.'
(Silva 2004, HVH 298)

\footnotetext{
${ }^{5}$ La co-ocurrencia de frases temporales que acotan el evento del tipo 'en / durante / por 5 minutos' también pueden distinguir eventos télicos (Моvет) de atélicos (Моvем); en yaqui, todas esas frases temporales se marcan con la posposición - po, por lo que no es posible explorar este tipo de estrategias.
} 
Guerrero: LA NATURALEZA DE LAS FRASES LOCATIVAS EN CONSTRUCCIONES DE...

$$
\begin{array}{llll}
\text { c. } \quad \text { Yookoria-po=ne } & \text { sikupuriam } & \text { jinu- } k & \text { [jita-nenenki-wa-po] } \\
\text { mañana-LOC=1sG.NOM } & \text { riñón.PL } & \text { comprar-PFV } & \text { cosa-RED.vender-PASS-LOC } \\
\text { 'En la mañana compré riñones en el mercado.' } &
\end{array}
$$

Esta misma posposición introduce al punto de origen de movimiento o transferencia. En los ejemplos de (7), la única interpretación posible para las frases locativas con - po es la de fuente, esto es, el cambio de lugar como consecuencia de una actividad previa. La interpretación de una locación estática, tiene poco sentido en estas construcciones:

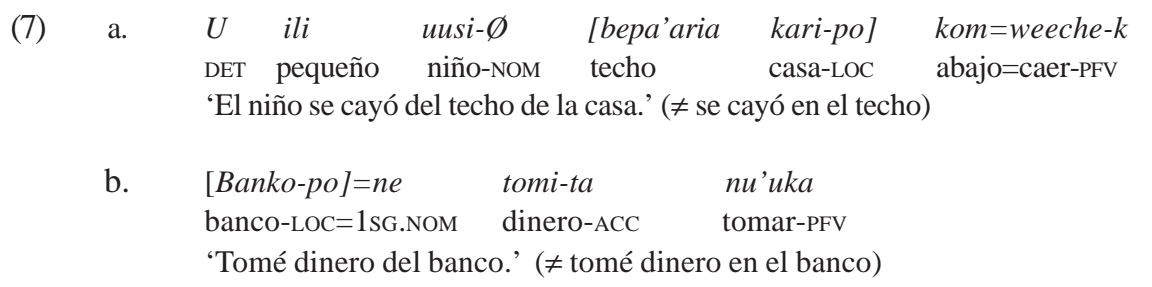

Ejemplos como los de (8a-b) podrían interpretarse como una locación dinámica (fuente) o estática (lugar), aunque los hablantes prefieren la primera lectura, ej. lo sacó del agua vs. lo sacó estando en el agua. Con menor frecuencia, el origen puede expresarse con la posposición betana y explicitar una relación de fuente-posesión 'desde, proveniente, tipo de' (8c); cuando se trata de una fuente humana, es posible que ocurra el direccional $-u$ en descripciones como la de (8d) para evitar la interpretación genitiva, ej. compré riñones del carnicero. 2sG.NOM pequeño niño-ACC agua-LOC salvar-PFV 'Tú salvaste el niño en/del agua.'
a. Empo ili usi-ta [ba'a-po] jinne'u-k
b. Inepo [Pesio-po] joome- $\varnothing$
1SG.NOM Hermosillo-LOC provenir-PRE
'Yo provengo de/radico en Hermosillo.'
c. Empo pannim un'upa-k [sa'aparia-betana]
2SG.NOM pan.PL traer-PFV Obregón-desde
'Tú trajiste pan de Obregón.'
d. $\quad$ Wakareo-ta-u] $=n e$
carnicero-ACC-DIR=1SG.NOM
$\begin{array}{ll}\text { sikupuriam } & \text { jinu- } k \\ \text { riñón.PL } & \text { comprar-PFV }\end{array}$
'Le compré riñones al carnicero.'

Con verbos que describen la posición o postura de la figura, una frase locativa marcada con -po necesariamente codifica locación estática (9a-b); con verbos que lexicalizan el movimiento de la figura, - $p o$ describe el punto de origen de la actividad (9c). 
a. $\begin{array}{ll}U & \text { chu'u- } \emptyset \\ \text { DET } & \text { perro-NOM }\end{array}$

'El perro está sentado afuera de la casa.'

b.
U kaba'i buki-Ø [kora-po]
DET caballo cria-NOM corral-LOC
weyek
'El becerrito está parado en el corral.'

\begin{tabular}{|c|c|c|}
\hline $\begin{array}{l}\text { Inepo } \\
\text { 1SG.NOM }\end{array}$ & $\begin{array}{l}\text { [Pesio-po] } \\
\text { Hermosillo-LOC }\end{array}$ & $\begin{array}{l}\text { [Vicam-meu] } \\
\text { Vicam-DIR.PL }\end{array}$ \\
\hline
\end{tabular}

pa'aku] katek

afuera sentado.sG

Ejemplos como los de (6) a (9) sugieren, por tanto, que el yaqui neutraliza la distinción entre lugar (relación espacial estática) y fuente (relación espacial dinámica), aunque distingue formalmente la codificación de meta (relación espacial dinámica). El sincretismo lugar $=$ fuente es uno de los patrones más raros, tipológicamente hablando, de acuerdo con los hallazgos reportados en Pantcheva (2010), por lo que bien vale la pena continuar la investigación de la codificación formal de las relaciones espaciales, no solo en yaqui sino también en las lenguas yutoaztecas más cercanas.

\section{VERBOS DE MOVIMIENTOENYAQUI}

Establecer la clasificación interna de los verbos de movimiento en lenguas particulares es una tarea compleja. La mayor parte de los autores coincide en dos grandes grupos: (i) verbos de movimiento o desplazamiento (MOVE) como ir, venir, salir, entrar, y (ii) verbos de manera de movimiento $\left(\mathrm{MOVE}_{\mathrm{M}}\right)$ como caminar, correr, nadar. Cada subgrupo puede, a su vez, sub-dividirse: el primero distingue eventos que solo perfilan el movimiento per se (ir, venir) de aquellos que lexicalizan el inicio o culminación del movimiento (entrar, salir), los que Beavers, Levin y Tham (2010) etiquetan como verbos direccionales o de trayectoria inherente (MоVе $)$; el segundo subgrupo puede dividirse en los que Jackendoff (1990) identifica como movimientos externos (caminar, correr, volar) y movimientos internos (bailar, tambalearse, inclinarse) del sujeto, sin implicaciones respecto a su locación o configuración con respecto a la base. En lenguas particulares, los equivalentes a estos tipos de verbos pueden asociarse a distintos grupos y/o sub-grupos, a partir de su comportamiento semántico y sintáctico de los componentes de la construcción. ${ }^{6}$

El yaqui tiende a seguir los patrones de lenguas/construcciones de marco-S, pues las raíces verbales lexicalizan, de manera preferencial, el movimiento y la manera, mientras que la trayectoria se expresa a través de satélites o deícticos espaciales. No obstante, también aparecen raíces direccionales. En comparación con otras lenguas del mismo tipo, el inventario léxico de verbos de movimiento en yaqui es relativamente pequeño.

${ }^{6}$ En la descripción, utilizo las formas léxicas del español (ir, correr, caminar, etc.) con fines descriptivos; esto no significa que las formas del yaqui y del español sean idénticas. Las traducciones fueron revisadas cuidadosamente con los hablantes de la lengua y reflejan, en la medida de lo posible, el equivalente más cercano. 
Antes de iniciar la descripción de los tipos de raíces verbales en yaqui, es necesaria una aclaración respecto a la codificación léxica de un subtipo de predicados. Los verbos de 'remoción' que perfilan de manera inherente el punto de origen de la actividad como salir y partir, y las versiones causativas remover, sacar, quitar, vaciar, se componen de un verbo de movimiento básico y el satélite yeu 'afuera'. Por ejemplo, siime expresa 'ir', mientras que yeu siime denota 'salir, irse, dejar un lugar'; wike expresa 'jalar, arrastrar' (10a) y la versión yeu wike codifica algo como 'sacar a rastras' (10b). Esto es, yeu deriva léxicamente los verbos de remoción; la expresión de la fuente codificada con -po suele co-ocurrir con este tipo de predicados. Sin embargo, la codificación de la fuente no está condicionada a la presencia de yeu, tal como se demuestra en los ejemplos de (10c-d).

$\begin{array}{llll}\text { (10) } & \text { Wakas-ta } & \text { [kora-po] } & \text { wike- } \varnothing \\ & \text { vaca-ACC } & \text { corral-LOC } & \text { jalar-PRE }\end{array}$

'Jala la vaca dentro del corral (muévela de lugar).'

b. Wame'e jamuch-im ball-ea [batwe-po] baba'ata

DEM.PL mujer-PL cubeta-INSTR río-LOC agua.CAUSA

'Esas mujeres acarrean agua desde el río con la cubeta.'

c. Sestul ta'a-po in kabaa [kora-po] yeu =wike-ka un día-LOC 1sG.GEN cabra corra-LOC fuera $=$ jarar-PFV 'Un día saqué a mis chivas del corral.' (Buitimea 2007; experiencia: 9)

d. Chikti ta'a-po [in jo'a-po]=ne [Torim-meu] weye siempre día-LOC $1 \mathrm{SG} . \mathrm{GEN}$ casa-LOC $=1 \mathrm{sG} . \mathrm{NOM}$ Torim-DIR.PL caminar.SG 'Todos los días camino de mi casa a Torim.'

\subsection{Verbos básicos de movimiento (MOVE)}

La raíz verbal que describe movimiento per se corresponde a siime 'ir' el cual hace uso de formas supletivas para número y aspecto (11). Verbos del tipo 'salir', 'bajar', 'subir' y 'venir' se forma a partir de la combinación de siime con los satélites yeu 'fuera', kom 'abajo', jika 'arriba' y algunos elementos deícticos o pro-adverbios (12).

(11) siime / saka

siika / sajak

(12) a. yeu=siime

b. kom=siime

c. jikau=siime

d. a'abo yeu=siime 'ir (no pasado, sg/pl)'

'ir (pasado, sg/pl)'

'salir (ir afuera).'

'bajar (ir abajo)'

'subir (ir arriba)'

'venir acá (ir afuera aquí)'

Del corpus general, 238 construcciones involucran una forma del verbo siime 'ir'. De ese grupo, 37\% (88/238) aparecen con el satélite yeu 'fuera' y corresponden, por tanto, a un verbo de remoción, i.e. 'irse, salir, partir'. En esos casos, yeu puede aparecer por sí solo (13a), o acompañarse de una frase que explicite la meta (13b), la fuente (13c) o ambos. 
Tabla 5. Construcciones con yeu siime 'salir' y tipos de locación

\begin{tabular}{|c|c|c|c|c|c|}
\hline \multicolumn{2}{|c|}{ raíz } & \multirow[b]{2}{*}{ yeu } & \multicolumn{2}{|c|}{ meta } & \multirow{2}{*}{$\begin{array}{c}\text { fuente } \\
\text { FN-po yeu }\end{array}$} \\
\hline & bal & & deíctico yeu & FN-u yeu & \\
\hline \multirow[t]{2}{*}{ siime } & 88 & 32 & 3 & 27 & 26 \\
\hline & $100 \%$ & $36 \%$ & & $64 \%$ & \\
\hline
\end{tabular}

Lo interesante aquí es que la ocurrencia del satélite yeu no es suficiente para completar la estructura conceptual de siime pues éste suele asociarse a frases locativas elaboradas que expliciten el punto culminante del movimiento. Nótese también que la combinación yeu siime 'salir' no se asocia a trayectorias atélicas introducidas con -bicha 'hasta, hacia'.
a. SanJoan
taewai-m-po, junama yeu=sasaka- $\varnothing$
San Juan día-PL-LOC siempre fuera=RED.ir.PL-PRE
'(Ellas) siempre salen el día de San Juan.' (Buitimea 2007; abuelo: 9)
b. In jaabi [Torim-meu] yeu=siime 1SG.GEN tío Torim-DIR.PL afuera=ir.SG.PRE 'Mi tío va a salir para Tórim.'
c. Inepo [Tosai kari-m-po] yeu=siime 1sG.NOM Blanca casa-PL-LOC afuera=ir.SG.PRE 'Yo me fui de Casas Blancas.'

El resto de las construcciones con el verbo siime (150/238) no hace uso de satélites (Tabla 6), por lo que la trayectoria del movimiento se establece a partir de distintas frases locativas, de manera preferencial, trayectorias télicas. Algunos ejemplos en (14).

Tabla 6. Combinaciones de la raíz verbal siime y tipos de locación

\begin{tabular}{|c|c|c|c|c|c|c|c|}
\hline \multirow{2}{*}{\multicolumn{2}{|c|}{$\begin{array}{c}\text { raíz } \\
\text { verbal }\end{array}$}} & \multirow[t]{2}{*}{$\varnothing$} & \multicolumn{2}{|c|}{ meta } & \multirow{2}{*}{$\begin{array}{l}\text { fuente } \\
\text { FN-po }\end{array}$} & \multicolumn{2}{|c|}{ ruta } \\
\hline & & & deíctico & $\mathrm{FN}-u$ & & FN-u-bicha & FN-u luula \\
\hline \multirow[t]{2}{*}{ siime } & 150 & 43 & 16 & 44 & 7 & 23 & 17 \\
\hline & $100 \%$ & $28 \%$ & & $45 \%$ & & \multicolumn{2}{|c|}{$27 \%$} \\
\hline
\end{tabular}
yooko $=n e$
mañana $=1$ sG.NOM
$\begin{array}{ll}{\left[a^{\prime} a b o\right]} & \text { siime- } \varnothing \\ \text { aquí } & \text { ir.SG-PRE }\end{array}$
'Mañana vengo temprano.'
b. $U$ achai Loreto- $\varnothing$ bea siika [costa-u-bicha] DET.NOM padre Loreto-NOM MD ir.SG.PFV Costa-DIR-HACIA 'Entonces el Sr. Loreto se fue hacia la Costa.'(Silva; Zorillo 5)
c. Junak bea $=$ te $\quad[$ pitaya $-u]=t e \quad k o m=s a j a-k$ luego MD=1PL.NOM pitahaya-DIR = 1PL.NOM abajo=ir.PL-PFV 'Luego entonces nos bajamos hacia La Pitahaya.' (Silva 2004; HVH 31)


Esto es, las construcciones de movimiento que involucran alguna forma del verbo siime se combinan de manera preferencial con trayectorias télicas que explicitan la meta o fuente del movimiento; aunque aparecen, las trayectorias atélicas son menos frecuentes $(27 \%)$.

\subsection{Verbos de movimiento y trayectoria $\left(\mathrm{MOVE}_{\mathrm{T}}\right)$}

Las raíces verbales que perfilan el punto de partida o llegada del movimiento incluyen noite 'regresar, volver', yepsa / yaja 'llegar' y kibake / kibacha 'entrar', además de watakte 'partir, alejarse', rukte 'aproximarse, arrimarse', naabujti 'pasar de largo', basu 'atravesar', ja'ати 'subir, montarse'; los datos de estos últimos verbos son muy pocos para establecer generalizaciones, por lo que el análisis parte de los ejemplos listados en (15). Al igual que el verbo semánticamente neutro siime, estos predicados pueden aparecer por sí solos (35\%), especificar la meta (55\%) y fuente (10\%), pero rara vez la vía o ruta $(2 \%)$.

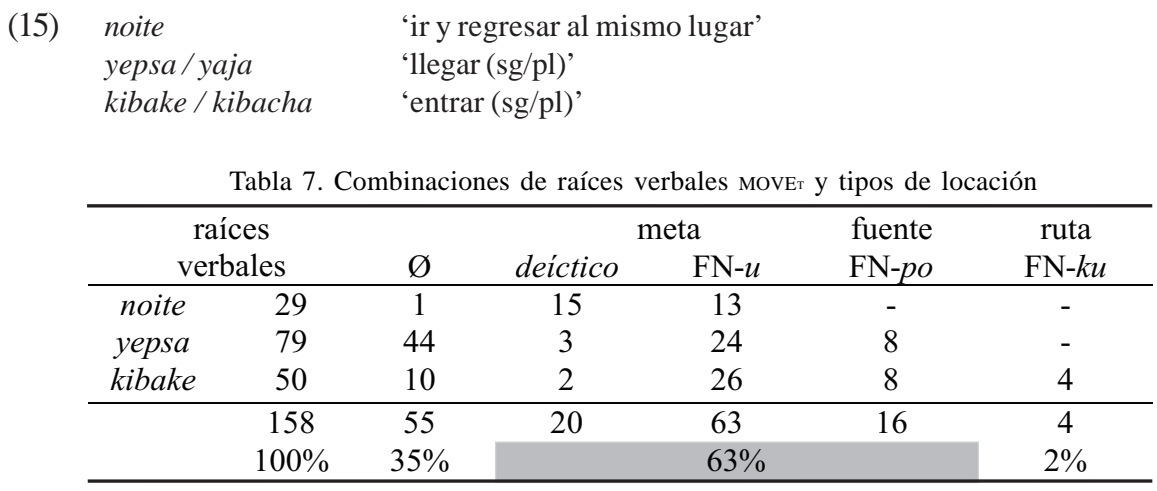

Como es de esperarse, este tipo de construcción resalta la descripción de una actividad télica asociada a metas o fuentes $(63 \%)$. La codificación de trayectorias atélicas apenas se observa con 'entrar' (4/50). En esos casos, la locación se marca con $-k u$ que describe algo como 'pasar a través de un hoyo, agujero (recinto cerrado)', como en (16d).
a.
$\begin{array}{ll}\text { Nak bea } & \text { yepsa- } k \\ \text { luego MD } & \text { llegar.sG-PFV }\end{array}$
ju'u
maala- $\varnothing$
'Entonces llegó la señora.' (Silva 2004; HVH:233)
b. $\quad\left[\mathrm{Jo}^{\prime} a-\mathrm{wa}-u\right]=$ ne $\quad$ yepsa-k
casa-GEN-DIR $=1$ SG.NOM llegar.SG-PFV
'Llegué a su casa.' (Buitimea 2007; achaques:31)

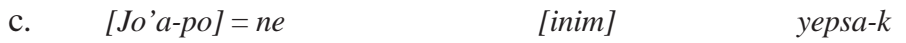
casa-LOC $=1$ sG.NOM aquí llegar.SG-PFV
'De mi casa llegué aquí.'



d. $U$
kukusaka-Ø
[gojo'o-ku]
kibake- $k$
DET
abeja-NOM
hOYO-DENTRO
entrar.SG-PFV

'La abeja peluquera se metió por en medio del hoyo.'

\subsection{Verbos de maneras externas de movimiento $\left(\mathrm{MOVE}_{\mathrm{M}}\right.$ externo)}

En contra de la predicción de Talmy y Slobin, el inventario de verbos que perfilan la manera del movimiento en yaqui es pequeño, y varios de sus miembros son formas compuestas del verbo básico siime. Las raíces básicas del tipo MOVE $_{M}$ se listan en (17) y las posibilidades de combinación con distintas frases locativas se resumen en la Tabla 8.

$$
\begin{array}{ll}
\text { bwite / tenne } & \text { 'correr }(\mathrm{sg} / \mathrm{pl}) ' \\
\text { weye / kaate } & \text { 'caminar }(\mathrm{sg} / \mathrm{pl}) ' \\
\text { weama / rejte } & \text { 'andar a pie }(\mathrm{sg} / \mathrm{pl})
\end{array}
$$

\begin{tabular}{|c|c|c|c|c|c|c|c|c|}
\hline & & & & meta & fuente & $\mathrm{ru}$ & & locación \\
\hline & ales & $\varnothing$ & deíctico & $\mathrm{FN}-u$ & FN-po & FN- $u$ & FN- $u$ & FN-po \\
\hline bwite & 55 & 30 & 4 & 12 & - & 7 & 4 & - \\
\hline weye & 97 & 18 & 38 & 16 & 5 & 7 & 12 & 2 \\
\hline weama & 40 & 3 & 8 & 4 & - & - & - & 22 \\
\hline & 192 & 51 & 50 & 32 & 5 & 14 & 16 & 24 \\
\hline & & & $26 \%$ & $17 \%$ & $2.5 \%$ & $7 \%$ & $8 \%$ & \\
\hline & $100 \%$ & $27 \%$ & & $45.5 \%$ & & & $15 \%$ & $12.5 \%$ \\
\hline
\end{tabular}

Tabla 8. Combinaciones de raíces verbales моvEм externas y tipos de locación

De nuevo, se observa una fuerte preferencia por especificar algún tipo de locación (63\%) sobre construcciones que no codifican dirección ni fondo (27\%). La primera diferencia que destaca entre raíces verbales MOVE у MOVЕ raíces MOVE $_{\mathrm{M}}$, por el otro, es la facilidad con que los últimos se asocian a deícticos espaciales (26\%) sobre frases que expliciten el tipo de trayectoria. Ahora bien, la predicción con respecto al primer grupo de verbos respecto al tipo de combinaciones locativas, era la asociación preferencial con frases que expliciten la meta, fuente, incluso sobre rumbo o dirección, situación que se cumple en yaqui, mientras que las observaciones tipológicas sobre verbos que perfilan la manera de movimiento sugieren que pueden, pero no necesita asociarse a ninguna locación en particular; si lo hacen, entonces las raíces моvе $_{\mathrm{M}}$ externas prefieren asociarse a locaciones estáticas. En yaqui, tales predicciones parecen no satisfacerse de manera clara, pues los equivalentes a 'correr' y 'caminar' no muestran ninguna preferencia. Algunos ejemplos con trayectorias télicas y atélicas:

a.

$\begin{array}{ll}\text { [Teta-m } & \text { beas] } \\ \text { piedra-PL } & \text { detrás } \\ \text { bweta } & \text { bea } \\ \text { pero } & \mathrm{MD}\end{array}$

euse-kan

ju-me'e esconderse-IMPFV DET-PL tenne- $k$ 'Estaban escondidos atrás de unas piedras, los carajos, pero huyeron.' 
GuERRERO: LA NATURALEZA DE LAS FRASES LOCATIVAS EN CONSTRUCCIONES DE...
b. Si'ime $=$ te $\quad$ Teta Kawi-u $]$ yeu=kat-ne todos $=1$ PL.NOM piedra $\quad$ cerro-DIR fuera=caminar.PL-POT
'Todos caminaremos al cerro Tetakawi'
c. Bwe $[$ teeka-u $]=$ ne noite $-k$
pues cielo-DIR $=1$ sG.NOM ir.venir-PFV
'Pues iba y venía al cielo.' (Buitimea 2007; sacerdote)

Contrario a las predicciones entorno a las raíces $\mathrm{MOVE}_{\mathrm{M}}$ destaca la poca ocurrencia de frases locativas que establecen la ubicación o lugar donde se lleva a cabo la actividad, i.e. locación estática. De los siete ejemplos con weye 'caminar', cinco de ellos se interpretan como la fuente del movimiento (19a-b) y los otros dos como lugar (19c).
a. O'ow-im into jaamuch-im [teopo-po] yeu=kate- $n$ hombre-PL y mujer-PL iglesia-LOC fuera=caminar.PL-PAS 'Hombres y mujeres salieron de la iglesia (lit. caminaron fuera)'
b. $U$ o'ou- $\varnothing \quad$ [jo'a-ро] [tеоро-и] weeye- $\varnothing$ DET hombre-LOC casa-LOC iglesia-DIR caminar.SG-PRE

'El hombre va de la casa a la iglesia'
c. [kuna-po waiwa] naa=weeye- $\varnothing$ cuna-LOC dentro lado.y.otro=caminar.SG-PRE '(El bebé) camina de un lado para el otro dentro de la cuna'

A diferencia de bwite 'correr' y weye 'caminar', la raíz weama 'andar a pie' es la única que muestra una alta preferencia por locaciones estáticas, esto es, movimiento en varias direcciones dentro de los límites de un lugar. En los ejemplos de (20) se expresa la ubicación del evento (pero no la fuente); una combinación como la de (20b) no es posible con este verbo.
a.

\subsection{Verbos de maneras internas de movimiento $\left(\mathrm{MOV}_{\mathrm{M}}\right.$ interno)}

Finalmente, los predicados que perfilan maneras internas de movimiento (o movimientos internos de la figura) del tipo waka'ate 'gatear', chitojte 'resbalarse', biakte 'rodar', ne'e 'volar', así como las formas ye'e 'bailar', ro'akte 'voltearse', tubukte 'saltar', chepte 'brincar', se asocian de manera casi exclusiva con locaciones estáticas y, en muy pocos casos (5/58), con elementos deícticos. En los ejemplos de (21), la frase locativa -po indica el lugar, el límite espacial en donde se lleva a cabo la acción; la ocurrencia de complementos direccionales quedan descartados. 


$\begin{array}{llllll}U & \text { ili } & \text { uusi- } \varnothing & \text { [bobo'o-po] } & (\text { *bobo'o- } u)^{\text {chepte- } k} \\ \text { DET } & \text { pequeño } & \text { niño-NOM } & \text { cama-LOC } & \text { cama-DIR } & \text { brincar-PFV }\end{array}$

Los verbos de tipo 'volar', 'flotar', 'arrastrarse' en yaqui son predicados compuestos que explicitan la manera y el movimiento, ej. ni ' $i$-sisime 'volar (volar-ir)', cha' $a$-sime 'flotar (colgar-ir)', wik-sime 'arrastrarse (jalar-moverse)'. Al contener mayor información léxica, estos predicados establecen distintas restricciones con respecto al tipo de locación. Por ejemplo, wiksime 'arrastrarse' puede elegir entre relaciones dinámicas de meta y fuente (22a), pero nunca locación estática; esta preferencia es similar a raíces MOvE. En cambio, ni'isisime y cha'assime no pueden asociarse a locaciones dinámicas y sólo se combinan con frases locativas estáticas marcadas con la posposición -po (22b), el patrón esperado para verbos MOVE . 'Las vacas fueron arrastradas hasta el corral (*en, dentro del corral).'

\begin{tabular}{|c|c|c|c|}
\hline $\begin{array}{l}\text { Kama- } \varnothing \\
\text { cocodrilo-NOM }\end{array}$ & $\begin{array}{l}\text { [batwe-po] } \\
\text { río-LOC }\end{array}$ & $\begin{array}{l}\text { (*batwe-u(-bicha) }) \\
\text { río-DIR-HACIA }\end{array}$ & $\begin{array}{l}\text { cha'asisime- } \varnothing \\
\text { flotar-PRE }\end{array}$ \\
\hline
\end{tabular}

\section{DISCUSIÓN}

A manera de resumen, la Tabla 9 muestra las combinaciones de tipos de raíces verbales y tipos de relaciones espaciales observadas en el corpus del yaqui. Aquí, la raíz verbal weama / rejte 'andar a pie' se incluye en el subgrupo de verbos de manera de movimiento interno, dada su fuerte preferencia a combinarse con locaciones estáticas, en lugar de relaciones espaciales dinámicas.

Tabla 9. Combinaciones de verbos de movimiento y tipos de locaciones

\begin{tabular}{lccccc}
\hline \multirow{2}{*}{$\begin{array}{c}\text { tipos de } \\
\text { raíces verbales }\end{array}$} & \multicolumn{4}{c}{$\begin{array}{c}\text { Relaciones Espaciales } \\
\text { dinámicas }\end{array}$} & estáticas \\
\cline { 2 - 6 } & & $\varnothing$ & trayectoria télica & trayectoria atélica & ubicación \\
\hline MOVE & 238 & 75 & 123 & 40 & - \\
MOVE $_{\mathrm{T}}$ & 158 & 55 & 99 & 4 & - \\
MOVE $_{\text {M EXTERNO }}$ & 152 & 48 & 75 & 30 & 2 \\
MOVE $_{\text {M INTERNO }}$ & 98 & 3 & 5 & - & 90 \\
\hline & 646 & 181 & 302 & 74 & 92
\end{tabular}


Un análisis basado en corpus, como el que aquí se presenta, permite corroborar la predicción de que lenguas/construcciones de marco-S describen la locación con bastante frecuencia y de manera elaborada, independientemente del tipo de raíz verbal, pues poco más del 70\% de las construcciones de movimiento codifica algún tipo de locación. Entre los tipos de raíces verbales que aparecen con más facilidad sin ningún tipo de complemento, son los del tipo моvе у моVЕт, mientras que las raíces MOVЕ $_{\mathrm{M}}$ internos evitan aparecer sin complementos. Nótese también que más de la mitad de las frases locativas introducen relaciones espaciales dinámicas, en particular, trayectorias télicas. Este es el patrón esperado para las raíces verbales que denotan movimiento per se (MOVE) y movimiento dirigido $\left(\mathrm{MOVE}_{\mathrm{T}}\right)$. Esta misma preferencia por explicitar metas y fuentes, sobre locaciones estáticas, evidencia los dos subtipos de raíces verbales моуЕе: entre aquellas que describen movimiento externo y se asocian fácilmente a relaciones dinámicas, y las que describen maneras de movimiento internas y prefieren asociarse con frases locativas de lugar.

Construcciones como las de (18) en donde algunos verbos de manera de movimiento se asocian con complementos direccionales son menos esperadas y, por tanto, planean varias interrogantes en torno a la correlación entre el tipo de verbo de movimiento (manera vs. dirección), el tipo de relación espacial (metas/fuentes, locaciones), y si se codifica o no la trayectoria del movimiento. En yaqui, verbos como 'correr' y 'caminar' no solo permiten sino que prefieren combinarse con frases direccionales dinámicas, lo que sugiere que estas raíces perfilan tanto la manera como la trayectoria del movimiento. En cambio, esas combinaciones están más restringidas con verbos del tipo volar, nadar, rodar, etc., que solo lexicalizan la manera del movimiento.

\section{COMENTARIOS FINALES}

El objetivo de este artículo fue explorar algunos aspectos de la estructura argumental de construcciones de movimiento en yaqui (yutoazteca, México). Con respecto a la codificación formal de las relaciones espaciales, se mostró que el yaqui neutraliza la distinción formal entre lugar y fuente, un patrón tipológico muy poco común. Se mostró también que, aunque el yaqui sigue varios de los patrones de lexicalización y combinación de constituyentes observados en lenguas de marco-S, la posibilidad de combinarse con locaciones dinámicas que delimiten temporal y aspectualmente el evento permite distinguir dos tipos de raíces que perfilan la manera del movimiento: los del tipo 'correr, caminar' que se comportan de manera similar a raíces verbales que perfilan el movimiento y la dirección, y los del tipo 'bailar', 'brincar', 'rodar', 'volar', 'andar' que se combinan exclusivamente con locaciones estáticas marcadas con -po. No obstante, la tendencia a expresar la culminación de un evento $\mathrm{MOVE}_{\mathrm{M}}$ con frases direccionales télicas es menos fuerte con 'correr' y 'caminar', en comparación con las raíces verbales del tipo MOVE у $\mathrm{MOVE}_{\mathrm{T}}$ en donde la ocurrencia de trayectorias atélicas es más restringida. 


\section{REFERENCIAS BIBLIOGRÁFICAS}

ASKE, Jon (1989). Path Predicates in English and Spanish: a closer look. Proceedings of Berkeley Linguistic Society 15: 1-14.

BEAVERS, John; Levin, Beth; Tham, Shiao Wei (2010). The Typology of Motion Events Revisited. Journal of Linguistics 46(2): 331-377.

BELLORO, Valeria; Guerrero, Lilián (2012). La interfaz gramática-discurso en las construcciones de movimiento en yaqui. Lingüística 27: 1-24.

.(2010). La función discursiva de los argumentos postpuestos en yaqui. En R. M. Ortiz Ciscomani (coord.). Estudios Lingüísticos, vol. 2: 71-95. Hermosillo: Unison.

BLAKE, Barry J. (1977). Case marking in Australian languages. Canberra: Australian Institute of Aboriginal Studies.

BOHNEMEYER, Jürgen; Pérez Báez, Gabriela (2008). Object to path in Mesoamerica: Semantic composition of locative and motion descriptions in Yucatec Maya and Juchitán Zapotec. Memoria del IX Encuentro Internacional de Lingüística en el Noroeste, vol. 2: 269-284. Hermosillo: UniSon.

BUITIMEA, Cresencio (2007). Preesio betana nottiwame. Hermosillo: Unison. Cifuentes-Férez, Paula (2009). A crosslinguistic study on the semantics of motion verbs in English and Spanish. Munich: Lincolm.

CIFUENTES HONRUBIA, José Luis (1999). Sintaxis y semántica del movimiento. Aspectos de gramática cognitiva. Alicante: Instituto de Cultura Juan Gil Albert.

CREGO GARCÍA, Ma. Victoria (2000). El complemento locativo en español: los verbos de movimiento y su combinatoria sintáctico-semántica. Santiago de Compostela: Universidad de Santiago de Compostela.

CUARTERO, Juan (2010). Estructuras argumentales de los verbos de desplazamiento del sujeto: una descripción del español frente a una descripción del inglés. The Bulletin of Hispanic Studies 87(2): 149-168.

ESTRADA, Zarina et al (2004). Diccionario Yaqui-Español y textos, obra de preservación lingüística. México: Plaza y Valdez.

FÉLIX, Rolando (n.f). Historia de vida CB (HVC). Manuscrito.

GUERRERO, Lilián (2004a). Verbos de movimiento y posición en yaqui. En Zarina Estrada; Z. A. Fernández; A. Álvarez (eds.). Estudios en lenguas amerindias. Homenaje a Ken Hale, pp. 199-222. Hermosillo: Unison.

.(2004b). ¿Intransitividad dividida en Yaqui? En I. Barreras; M. Castro (eds.). Memorias del VII Encuentro Internacional de Lingüística en el Noroeste, Vol. 1, pp. 335-367. Hermosillo: Unison. ; Belloro, Valeria A. (en prep.). Sintaxis y pragmática de los complementos locativos en yaqui y náhuatl. En L. Guerrero (ed.), Verbos de movimiento en lenguas de América. México: UNAM.

GUTIÉRREZ, Paola (2011). Predicados supletivos de posición y plurales léxicos en la lengua yaqui. Tesis de licenciatura, Escuela Nacional de Antropología e Historia.

IBAÑEZ, Sergio (2005). Los verbos de movimiento intransitivos en español. México: UNAM/UNAH.

IBARREXTE-ANTUÑO, Iraide (2004). Dicotomías frente a continuos en la lexicalización de los eventos de movimiento. Revista española de lingüística 34(2): 481-510. 
JACKENDOFF, Ray (1990). Semantic Structures. Cambridge: MIT Press.

LAMIROY, Béatrice (1991). Léxico y gramática del español. Estructuras verbales de espacio y de tiempo. Barcelona: Anthropos.

MINTZ, Malcolm W. (1994). A student's grammar of Malay and Indonesian. Perth: Indonesian /Malay Texts and Resources.

MORIMOTO, Yuko (2000). Verbos de movimiento. Madrid: Visor. .(2007) Grammar of 'manner of motion' verbs in English and Spanish: between lexicon and syntax. En N. Delbecque; B. Cornillie (eds.), On Interpreting Construction Schemas: from Action and Motion to Transitivity and Causality, pp. 287-305. Berlín: Mouton de Gruyter.

NIKITINA, Tatiana (2009). Subcategorization pattern and lexical meaning of motion verbs: a study of the source/goal ambiguity. Linguistics 47(5): 1113-1141.

NOONAN, Michael (2008). Patterns of development, patterns of syncretism of relational morphology in the Bodic languages. En Jóhanna Barðdal; Shobhanna L. Chelliah (eds.). The role of semantics and pragmatics in the development of case, pp. 261-281. Amsterdam \& Philadelphia: John Benjamins.

PANTCHEVA, Marina. (2010). The syntactic structure of Location, Goals and Sources. Linguistics 48(5): $1043-81$.

RICE, Sally; KaorI Kabata (2007). Crosslinguistic grammaticalization patterns of the allative. Linguistic Typology 11. 451-514.

SILVA E., Carlos (2004). La secuencia temporal en el discurso narrativo Yaqui, anexo Historia de Vida de Don Hilario (HVH). Tesis de Maestría. Hermosillo, Sonora: Universidad de Sonora.

SILVA E. Carlos et al (1997). Jiak nokpo etejoim, Pláticas en lengua yaqui. Hermosillo: Unison.

SLOBIN, Dan I. (1996). Two ways to travel: Verbs of motion in English and Spanish. En Sandra A.Thompson; Masayoshi Shibatani (eds.). Grammatical Constructions: Their Form and Meaning, pp. 195-217. Oxford: Oxford University Press.

.(2004). The many ways to search for a frog: Linguistic typology and the expression of motion events. En Sven Strömqvist y Ludo Verhoeven (eds.). Relating Events in Narrative: vol. 2. Typological and contextual perspectives, pp. 219-257. Mahwah, NJ: Lawrence Erlbaum Associates.

TALMY, Leonard (1985). Lexicalization patterns: semantic structure in lexical forms. En Timothy Shopen (ed.). Language Typology and Syntactic Description, III, pp. 57-149. Cambridge: Cambridge University Press.

(1991). Path to realization: a typology of event conflation. En Papers of the $17^{\text {th }}$ Annual Meeting of the Berkeley Linguistics Society, pp. 480-520. Berkeley: Berkeley Linguistics Society. (2000). Toward a Cognitive Semantics, 2 volúmenes. Cambridge: MIT Press.

TERNES, Elmar (1992). The Breton language. En Donald Macaulay (ed.). The Celtic languages, pp. 371-452. Cambridge: Cambridge University Press.

TESNIÈRE, Lucien (1959). Éléments de syntaxe structurale. Paris: Klincksieck.

Recebido 10/4/2012

Versão revista 14/5/2012

Aceito 30/5/2012. 